\title{
Comparison of single injection and three monthly injections of intravitreal bevacizumab for macular edema associated with branch retinal
} vein occlusion

\author{
This article was published in the following Dove Press journal: \\ Clinical Ophthalmology \\ 23 January 2015 \\ Number of times this article has been viewed
}

\section{Yuka Ito \\ Yoshitsugu Saishin \\ Osamu Sawada \\ Masashi Kakinoki \\ Taichiro Miyake \\ Tomoko Sawada \\ Hajime Kawamura \\ Masahito Ohji}

Department of Ophthalmology, Shiga University of Medical Science, Otsu, Shiga, Japan
Correspondence: Yoshitsugu Saishin Department of Ophthalmology,

Shiga University of Medical Science,

Seta-Tsukinowacho, Otsu,

Shiga 520-2192, Japan

Tel +8I 775482276

Fax +8I 775482279

Email saishin@belle.shiga-med.ac.jp
Purpose: Our aim was to compare the 1 year efficacy and safety results of intravitreal bevacizumab (IVB) in two prospective, consecutive groups of patients with macular edema (ME) following branch retinal vein occlusion (BRVO).

Patients and methods: Twenty-five eyes with ME after BRVO received one IVB injection (single-injection group) and 27 eyes received three monthly IVB injections (three-injection group). Both groups were followed monthly for 12 months. The best-corrected visual acuity (BCVA) and the central foveal thickness (CFT) on optical coherence tomography were evaluated before and after treatment. Patients were eligible to receive an IVB injection if the mean CFT increased $100 \mu \mathrm{m}$ or more or the BCVA decreased 0.1 logarithm of the minimum angle of resolution (logMAR) unit or more compared with values measured on the last visit.

Results: The mean logMAR BCVA and CFT, respectively, improved from 0.56 to 0.33 and from $598 \mu \mathrm{m}$ to $348 \mu \mathrm{m}$ in the single-injection group $(P<0.001)$ and from 0.55 to 0.26 and from $514 \mu \mathrm{m}$ to $293 \mu \mathrm{m}$ in the three-injection group $(P<0.001)$. During the study period, the mean total number of injections was significantly smaller in the single-injection group than in the three-injection group ( 2.1 and 4.3 , respectively, $P<0.001$ ). No serious complications related to the IVB injections developed in either group.

Conclusion: The single-injection group achieved similar visual outcomes for ME secondary to BRVO with fewer injections compared with the three-injection group.

Keywords: branch retinal vein occlusion, bevacizumab, single intravitreal injection, three monthly intravitreal injections

\section{Introduction}

Retinal occlusive disorders are second in prevalence after diabetic retinopathy. ${ }^{1}$ Macular edema (ME) associated with branch retinal vein occlusion (BRVO) is a common complication. ${ }^{2}$ Although a study reported that macular grid-pattern laser photocoagulation improved visual acuity (VA), most patients had limited improvement in VA in other studies. ${ }^{2-5}$ Some studies have reported that intravitreal injection of triamcinolone acetonide decreased ME, but this treatment frequently caused adverse effects. ${ }^{1,2,6,7}$

Intravitreal bevacizumab (IVB) injections have been reported to be effective. ${ }^{8-11}$ However, IVB injection is an off-label treatment and there are no specific recommendations regarding the frequency of injection for effective long-term results.

We studied two prospective, consecutive groups of patients with ME following BRVO. One group received a single IVB injection, the other received three monthly IVB injections, and the 1 year efficacy and safety results were compared in the two groups. 


\section{Patients and methods}

\section{Inclusion criteria}

Patients with ME after BRVO were included. Patients had a central foveal thickness (CFT) exceeding $300 \mu \mathrm{m}$ on optical coherence tomography (OCT; Cirrus HD-OCT, Carl Zeiss Meditec AG, Jena, Germany) and a decimal VA of 0.5 or worse.

\section{Exclusion criteria}

Eyes with glaucoma, diabetic retinopathy, and inflammatory disease were excluded. Patients with a recent history of stroke or myocardial infarction, unstable angina pectoris, uncontrolled hypertension, uncompensated renal insufficiency, pregnancy, prior anti-vascular endothelial growth factor (VEGF) treatment or allergy to bevacizumab were also excluded.

\section{Re-injection criteria}

Patients were eligible to receive an IVB injection if there was an increase in the mean CFT of $100 \mu \mathrm{m}$ or more or a decrease in the BCVA of 0.1 logarithm of the minimum angle of resolution $(\log \mathrm{MAR})$ unit or more compared with values measured on the last visit.

\section{Single-injection group}

We prospectively analyzed 25 consecutive eyes of 25 patients (nine males and 16 females) with ME following BRVO who were enrolled from August 2007 to March 2009 at the Shiga University of Medical Science Hospital. The Institutional Review Board of the hospital approved the study protocol. Patients received one IVB injection at baseline and no additional treatments were performed within 3 months of the initial injection (month 1 and month 2). IVB was administered as needed if the pre-specified criteria were met during months 3 to 11 (referred to as the pro re nata [PRN] period). Four patients received prior treatment with photocoagulation (three patients) or posterior sub-tenon injection of triamcinolone acetonide (one patient) more than 9 months prior to the first IVB injection.

\section{Three-injection group}

We prospectively consecutively studied 27 eyes of 27 patients (15 males and 12 females) with ME after BRVO who were enrolled from December 2009 to November 2010 at the Shiga University of Medical Science Hospital. The Institutional Review Board of the Shiga University of Medical Science Hospital approved the study protocol. Patients received three monthly IVB injections (day $0-$ month 2). IVB was then administered as needed if pre-specified criteria were met during the PRN period. Two patients received prior treatment with photocoagulation (one patient) or posterior sub-tenon injection of triamcinolone acetonide (one patient) more than 5 months prior to the first IVB injection.

\section{Study visits and assessments}

Patients were examined at baseline (day 0), week 1, and then monthly from months 1 to 12 . At each visit, patients underwent a complete ocular examination. The BCVA was expressed as decimal VA using a Landolt $\mathrm{C}$ chart and converted to $\log$ MAR VA for analysis. The CFT was measured using the macular cube $512 \times 128$ mode protocol and defined as the central area within a diameter of $1 \mathrm{~mm}$ on Cirrus HDOCT images.

\section{Statistical analysis}

Data were analyzed using SPSS II for Windows (SPSS Inc., Chicago, IL, USA). Differences in the logMAR BCVA and CFT between baseline and 12 months after the first injection were analyzed using a paired samples $t$-test. Differences in the changes in the logMAR BCVA and CFT between the two treatment groups were analyzed using a two-sample Student's $t$-test. A $P$-value less than 0.05 was considered to be statistically significant.

A chi-square test was used to compare the difference in percentage of patients who gained $0.3 \log$ MAR units or more in BCVA between the two groups.

\section{Results}

\section{Baseline characteristics}

The baseline characteristics are shown in Table 1. The mean ( \pm standard deviation) age was $69.3 \pm 7.8$ years (range 55-81) in the single-injection group and 68.0 \pm 10.7 years (range 44-88) in the three-injection group. There were no significant differences between the groups in gender, age, BCVA, or CFT.

\section{Duration of BRVO before the treatment}

There was no significant difference in mean interval between the onset of BRVO and the first intravitreal injection of bevacizumab $(3.57 \pm 2.87$ months [range 1-11] in the singleinjection group versus (vs) $3.73 \pm 3.03$ months [range 1-12] in the three-injection group; $P=0.85)$.

\section{VA}

The mean logMAR BCVA values are shown in Figure 1. There was no significant difference in the mean $\log$ MAR 
Table I Baseline patient characteristics in the two treatment groups

\begin{tabular}{lll}
\hline Characteristic & $\begin{array}{l}\text { Three IVB } \\
\text { group }(\mathbf{n}=\mathbf{2 7})\end{array}$ & $\begin{array}{l}\text { Single IVB } \\
\text { group }(\mathbf{n}=\mathbf{2 5})\end{array}$ \\
\hline No patients & 27 & 25 \\
Female/male (eyes) & $12 / 15$ & $16 / 9$ \\
Age (years, mean \pm SD) & $68.0 \pm 10.7$ & $69.32 \pm 7.8$ \\
BCVA (logMAR, mean \pm SD) & $0.55 \pm 0.24$ & $0.56 \pm 0.22$ \\
CFT $(\mu \mathrm{m}$, mean \pm SD) & $514 \pm 172$ & $598 \pm 186$ \\
\hline
\end{tabular}

Abbreviations: IVB, intravitreal bevacizumab; BCVA, best-corrected visual acuity; CFT, central foveal thickness; logMAR, logarithm of the minimum angle of resolution; SD, standard deviation.

BCVA at baseline $(0.56 \pm 0.22$ [range $0.30-1.15$ ] in the single-injection group vs $0.55 \pm 0.24$ [range $0.30-1.05$ ] in the three-injection group; $P=0.84$ ). In the single-injection group, the mean logMAR BCVA improved from $0.56 \pm 0.22$ (range 0.30-1.15) at baseline to $0.47 \pm 0.26$ (range 0-1.05), $0.42 \pm 0.33$ (range $0-1.15$ ), $0.39 \pm 0.27$ (range $0.05-1.05$ ), and $0.33 \pm 0.29$ (range, $-0.18-1.05$ ) at months $1,3,6$, and 12 , respectively, and significantly from 0.56 at baseline to 0.33 at month $12(P<0.001)$.

In the three-injection group, the mean baseline $\log M A R$ BCVA improved from $0.55 \pm 0.24$ (range 0.30-1.05) to $0.40 \pm 0.24$ (range $0-1.00$ ), $0.32 \pm 0.22$ (range $-0.07-0.82$ ), $0.30 \pm 0.22$ (range $0-0.70$ ) and $0.26 \pm 0.25$ (range $-0.18-0.70$ ) at months $1,3,6$, and 12 , respectively, and significantly from 0.55 at baseline to 0.26 at month $12(P<0.001)$. The mean $\log$ MAR BCVA did not differ significantly at month 12 between the two groups $(P=0.389)$.

The BCVA improved by $0.3 \log$ MAR units or more at month 12 in nine (36\%) eyes, remained stable in $14(56 \%)$

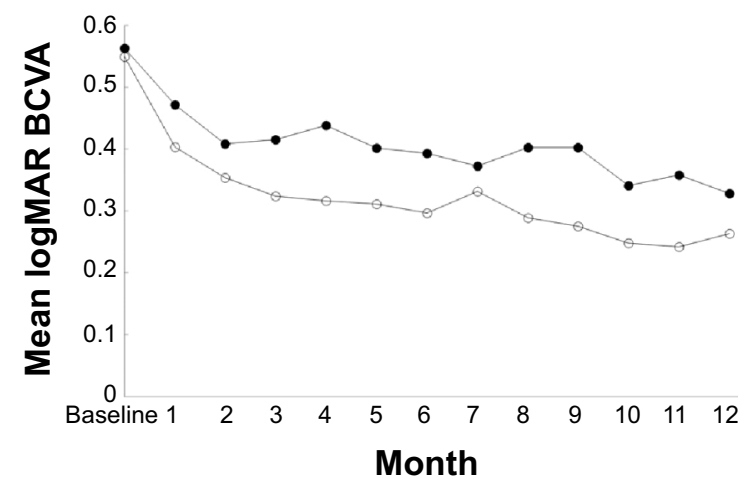

Figure I The change in the mean logarithm of the minimum angle of resolution best-corrected visual acuity (logMAR BCVA) from baseline to month 12 .

Notes: In the one-injection group (solid circles), the mean logMAR BCVA improved slightly from 0.56 at baseline to 0.47 at month I $(P=0.04)$ and improved significantly to 0.33 at month $12(P<0.00 \mathrm{I})$. In the three-injection group (open circles), the mean logMAR BCVA improved further to 0.40 at month I $(P<0.00$ I) and improved significantly from 0.55 at baseline to 0.26 at month $12(P<0.00 I)$. There was no significant betweengroup difference in the mean logMAR BCVA at month 12 ( $P=0.389)$. eyes, and worsened by $0.3 \log$ MAR units or more in two $(8 \%)$ eyes in the single-injection group, and improved by $0.3 \mathrm{log}$ MAR units or more at month 12 in $16(59 \%)$ eyes, remained stable in ten (37\%) eyes, and worsened by $0.3 \log$ MAR units or more in one (4\%) eye in the three-injection group. There was no between-group difference in the percentage of eyes with improved BCVA $(P=0.093)$ or in the percentage of eyes with worsened $\operatorname{logMAR}$ BCVA $(P=0.603)$.

\section{CFT}

The mean CFT is shown in Figure 2. In the single-injection group, the mean \pm standard deviation CFT decreased from $598 \pm 186 \mu \mathrm{m}$ at baseline to $347 \pm 133,476 \pm 139,384 \pm 141$, and $348 \pm 134 \mu \mathrm{m}$ at months $1,3,6$, and 12 , respectively, and decreased significantly $(P<0.001)$ from $598 \pm 186 \mu \mathrm{m}$ at baseline to $348 \pm 134 \mu \mathrm{m}$ at month 12 . In the three-injection group, the mean CFT decreased significantly $(P<0.001)$ from $514 \pm 172 \mu \mathrm{m}$ at baseline to $277 \pm 57,256 \pm 65,322 \pm 114$, and $293 \pm 102 \mu \mathrm{m}$ at months $1,3,6$, and 12 , respectively. The mean CFT at month 12 did not differ significantly between the two groups $(P=0.101)$.

\section{Number of additional injections}

During the PRN period, additional injections were required in $19(76 \%)$ of 25 eyes in the single-injection group (one injection in eleven eyes, two injections in seven eyes, and three injections in one eye) and $16(59 \%)$ of 27 eyes in the threeinjection group (one injection in four eyes, two injections in seven eyes, three injections in three eyes, four injections in one eye, and five injections in one eye). The mean number of additional injections was $1.1 \pm 0.8$ in the single-injection

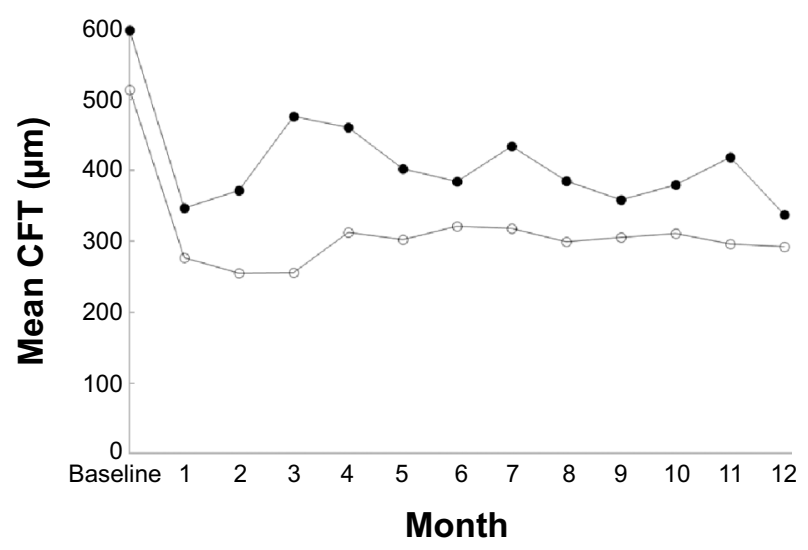

Figure 2 The mean change in central foveal thickness (CFT) from baseline to month 12 . Notes: The mean CFT decreased significantly from $598 \pm 186 \mu \mathrm{m}$ at baseline to $348 \pm 134 \mu \mathrm{m}$ at month 12 in the one-injection group (solid circles; $P<0.00 \mathrm{I}$ ) and from $514 \pm 172 \mu \mathrm{m}$ at baseline to $293 \pm 102 \mu \mathrm{m}$ at month 12 in the three-injection group (open circles; $P<0.00 \mathrm{I}$ ). There was no significant between-group difference in the mean CFT at month $12(P=0.101)$. 
group and 1.3 \pm 1.4 in the three-injection group. The number of additional injections did not differ significantly $(P=0.507)$ between the two groups.

During the study period, the mean total number of injections was significantly smaller in the single-injection group than in the three-injection group $(2.1 \pm 0.8$ vs $4.3 \pm 1.4$; $P<0.001)$.

\section{Complications}

No serious complications related to the IVB injections developed in either group.

\section{Discussion}

Previous studies have reported the efficacy of IVB for ME due to BRVO. However, few studies have reported the long-term efficacy of three monthly IVB injections and to our best knowledge, no studies have reported the results of treatment with single IVB injections and three monthly IVB injections in two prospective, consecutive groups of patients with ME following BRVO and compared the 1 year efficacy and safety results between these groups. A total of 52 patients were enrolled in the study, ie, 25 consecutive eyes of 25 patients initially treated with one IVB injection followed by PRN treatment and 27 consecutive eyes of 27 patients initially treated with three monthly IVB injections followed by PRN treatment.

The mean $\log$ MAR BCVA improved significantly from 0.56 at baseline to 0.33 at month 12 in the single injection group $(P<0.001)$ and from 0.55 at baseline to 0.26 at month 12 in the three-injection group $(P<0.001)$. The current results were similar to those of previous studies (Table 2). ${ }^{12-15}$ Comparing the results among different studies is difficult because of the variations in inclusion criteria and different retreatment criteria. In the current study, 1 year efficacy and safety results were compared between two groups of patients with ME after BRVO (a single IVB injection group and three monthly IVB injection group).
The groups were comparable because we prospectively used the same inclusion criteria, the same retreatment criteria, and the same follow-up schedule except for the loading phase. Patients in the two groups were not randomized. However, we believe that the likelihood of sample selection bias was small because consecutive patients in the single-injection group were enrolled from August 2007 to March 2009, and consecutive patients in the three-injection group were enrolled from December 2009 to November 2010. After comparing the results, there was no significant $(P=0.389)$ difference between the two groups regarding improvement in the logMAR BCVA.

Consistent with previous findings (Table 2), ${ }^{12-15}$ we found that the mean CFT decreased significantly from $598 \mu \mathrm{m}$ at baseline to $348 \mu \mathrm{m}$ at month 12 in the single-injection group $(P<0.001)$ and from $514 \mu \mathrm{m}$ at baseline to $293 \mu \mathrm{m}$ at month 12 in the three-injection group $(P<0.001)$. There was no significant $(P=0.101)$ between-group difference in CFT improvement.

Krohne et al reported that drug elimination from the aqueous humor closely parallels that from the vitreous and concluded that the aqueous half-life of $1.5 \mathrm{mg}$ of intravitreally injected bevacizumab in humans is 9.82 days. ${ }^{16}$ Miyake et al reported that the half-life of $1.25 \mathrm{mg}$ of intravitreally injected bevacizumab in macaque eyes is 2.8 days in the aqueous humor. ${ }^{17}$ The half-life of bevacizumab is therefore short and retreatment is often required. ${ }^{16-18}$ In the current study, the average number of additional injections was 1.1 in the single-injection group, and re-injections were performed in 19 (76\%) eyes during the PRN period. In the three-injection group, the average number of additional injections was 1.3, and re-injections were performed in 16 (59\%) eyes during the PRN period. Comparison of results of IVB injection at 12 months between previous studies and the current study is shown in Table 2.

It is difficult to compare the mean number of additional injections among different studies because of inter-study

Table 2 Comparison of results of intravitreal injection of bevacizumab at 12 months between previous studies and the current study

\begin{tabular}{|c|c|c|c|c|c|}
\hline Study & $\begin{array}{l}\text { Current study } \\
\text { single injection }\end{array}$ & Jaissle et $\mathrm{al}^{12}$ & Kondo et $\mathrm{al}^{13}$ & $\begin{array}{l}\text { Current study three } \\
\text { monthly injections }\end{array}$ & Demir et al $^{15}$ \\
\hline Eyes & 25 & 23 & 50 & 27 & 33 \\
\hline Injections & Single & Single & Single & Three monthly & Three monthly \\
\hline Baseline BCVA & 0.56 & 0.50 & 0.53 & 0.55 & 0.66 \\
\hline BCVA at 12 months & 0.33 & 0.20 & 0.26 & 0.26 & 0.22 \\
\hline Baseline CFT & 598 & 395 & 523 & 514 & 494 \\
\hline CFT at 12 months & 348 & 255 & 305 & 293 & 262 \\
\hline Additional injections & 1.1 & 2.4 & 1.0 & 1.3 & 2.3 \\
\hline
\end{tabular}

Abbreviations: BCVA, best-corrected visual acuity; CFT, central foveal thickness. 
variation in inclusion criteria and retreatment criteria. In the current study, the mean number of additional injections was compared between the single-injection group and the threeinjection group because the same inclusion and retreatment criteria were used prospectively, and no significant betweengroup difference in the number of additional injections was shown $(P=0.507)$.

The mean total number of injections was smaller in the single-injection group than the three-injection group $(2.1 \pm 0.8$ vs $4.3 \pm 1.4$, respectively) because the mean number of additional injections was similar between the two groups. No serious complications developed in either group. Therefore, the single injection protocol was safer and less invasive than the three-injection one.

The mean CFT in the single-injection group increased at months 2 and 3 and retreatment guidelines prohibited additional injections. There was no significant difference in the $\log$ MAR BCVA between the two groups at months 2 and 3 ( $P=0.403$ and $P=0.244$, respectively). Increases in the CFT in the short term may not have an immediate effect on BCVA, whereas increases in the CFT in the long term may result in worsening of the BCVA. ${ }^{19}$

The absence of a significant between-group difference in the mean CFT and the logMAR BCVA at month 12 in patients with ME after BRVO indicated that one IVB injection followed by PRN treatment had similar effectiveness to three monthly IVB injections followed by PRN treatment in improving BCVA and CFT. Furthermore, the total number of injections was smaller in the single-injection group than in the three-injection group.

No complications occurred in our two study groups, but severe side effects occurred in other studies. Minimizing the total number of injections reduces the risk of complications associated with IVB.

Although circulating VEGF protects the integrity and patency of vessels, prolonged anti-VEGF treatment can increase the risk of thromboembolic events, ${ }^{20-22}$ and reduced number of injections can lower the risk of serious adverse events.

Notably, one limitation of our study was the relatively small number of patients with available imaging, possibly preventing our analysis from reaching statistical significance.

We showed that similar visual outcomes for ME secondary to BRVO may be achieved with fewer injections when one IVB injection is followed by PRN treatment rather than when three IVB injections are followed by PRN treatment; however, a prospective, double-masked, randomized study with a large number of patients is warranted.

\section{Disclosure}

Supported in part by a grant from the Ministry of Education, Culture, Sports, Science and Technology of Japan (\#24592668). The authors have no conflicts of interest to disclose.

\section{References}

1. Cakir M, Dogan M, Bayraktar Z, et al. Efficacy of intravitreal triamcinolone for the treatment of macular edema secondary to branch retinal vein occlusion in eyes with or without grid laser photocoagulation. Retina. 2008;28(3):465-472.

2. Rehak J, Rehak M. Branch retinal vein occlusion: Pathogenesis, visual prognosis, and treatment modalities. Curr Eye Res. 2008;33(2):111-131.

3. No authors listed. Argon laser photocoagulation for macular edema in branch vein occlusion. The branch vein occlusion study group. Am J Ophthalmol. 1984;98(3):271-282.

4. Battaglia Parodi M, Saviano S, Ravalico G. Grid laser treatment in macular branch retinal vein occlusion. Graefes Arch Clin Exp Ophthalmol. 1999;237(12):1024-1027.

5. Battaglia Parodi M, Saviano S, Bergamini L, Ravalico G. Grid laser treatment of macular edema in macular branch retinal vein occlusion Doc Ophthalmol. 1999;97(3-4):427-431.

6. Cekic O, Chang S, Tseng JJ, et al. Intravitreal triamcinolone injection for treatment of macular edema secondary to branch retinal vein occlusion. Retina. 2005;25(7):851-855

7. Higashiyama T, Sawada O, Kakinoki M, Sawada T, Kawamura H, Ohji M. Prospective comparisons of intravitreal injections of triamcinolone acetonide and bevacizumab for macular oedema due to branch retinal vein occlusion. Acta Ophthalmol. 2013;91(4):318-324.

8. Rabena MD, Pieramici DJ, Castellarin AA, Nasir MA, Avery RL. Intravitreal bevacizumab (avastin) in the treatment of macular edema secondary to branch retinal vein occlusion. Retina. 2007;27(4):419-425.

9. Gunduz K, Bakri SJ. Intravitreal bevacizumab for macular oedema secondary to branch retinal vein occlusion. Eye (Lond) 2008;22(9):1168-1171.

10. Moradian S, Faghihi H, Sadeghi B, et al. Intravitreal bevacizumab vs Sham treatment in acute branch retinal vein occlusion with macular edema: Results at 3 months (report 1). Graefes Arch Clin Exp Ophthalmol. 2011;249(2):193-200.

11. Yunoki T, Miyakoshi A, Nakamura T, Fujita K, Fuchizawa C, Hayashi A. Treatment of macular edema due to branch retinal vein occlusion with single or multiple intravitreal injections of bevacizumab. Jpn J Ophthalmol. 2012;56(2):159-164.

12. Jaissle GB, Leitritz M, Gelisken F, Ziemssen F, Bartz-Schmidt KU, Szurman P. One-year results after intravitreal bevacizumab therapy for macular edema secondary to branch retinal vein occlusion. Graefes Arch Clin Exp Ophthalmol. 2009;247(1):27-33.

13. Kondo M, Kondo N, Ito Y, et al. Intravitreal injection of bevacizumab for macular edema secondary to branch retinal vein occlusion: Results after 12 months and multiple regression analysis. Retina. 2009; 29(9):1242-1248.

14. Byun YJ, Roh MI, Lee SC, Koh HJ. Intravitreal triamcinolone acetonide versus bevacizumab therapy for macular edema associated with branch retinal vein occlusion. Graefes Arch Clin Exp Ophthalmol. 2010;248(7):963-971

15. Demir M, Oba E, Gulkilik G, Odabasi M, Ozdal E. Intravitreal bevacizumab for macular edema due to branch retinal vein occlusion: 12-month results. Clin Ophthalmol. 2011;5:745-749.

16. Krohne TU, Eter N, Holz FG, Meyer CH. Intraocular pharmacokinetics of bevacizumab after a single intravitreal injection in humans. Am J Ophthalmol. 2008;146(4):508-512.

17. Miyake T, Sawada O, Kakinoki M, et al. Pharmacokinetics of bevacizumab and its effect on vascular endothelial growth factor after intravitreal injection of bevacizumab in macaque eyes. Invest Ophthalmol Vis Sci. 2010;51(3):1606-1608. 
18. Bakri SJ, Snyder MR, Reid JM, Pulido JS, Singh RJ. Pharmacokinetics of intravitreal bevacizumab (avastin). Ophthalmology. 2007;114(5):855-859.

19. Heier JS, Brown DM, Chong V, et al. Intravitreal aflibercept (vegftrap-eye) in wet age-related macular degeneration. Ophthalmology. 2012;119(2): 2537-2548.

20. Costagliola C, Agnifili L, Arcidiacono B, et al. Systemic thromboembolic adverse events in patients treated with intravitreal anti-VEGF drugs for neovascular age-related macular degeneration. Expert Opin Biol Ther. 2012;12(10):1299-1313.
21. Semeraro F, Morescalchi F, Duse S, Gambicorti E, Romano MR, Costagliola C. Systemic thromboembolic adverse events in patients treated with intravitreal anti-VEGF drugs for neovascular age-related macular degeneration: an overview. Expert Opin Drug Saf. 2014; 13(6):785-802.

22. Schmid MK, Bachmann LM, Fäs L, Kessels AG, Job OM, Thiel MA. Efficacy and adverse events of aflibercept, ranibizumab and bevacizumab in age-related macular degeneration: a trade-off analysis. Br J Ophthalmol. Epub 2014 Jun 11.

\section{Publish your work in this journal}

Clinical Ophthalmology is an international, peer-reviewed journal covering all subspecialties within ophthalmology. Key topics include: Optometry; Visual science; Pharmacology and drug therapy in eye diseases; Basic Sciences; Primary and Secondary eye care; Patient Safety and Quality of Care Improvements. This journal is indexed on

\section{Dovepress}

PubMed Central and CAS, and is the official journal of The Society of Clinical Ophthalmology (SCO). The manuscript management system is completely online and includes a very quick and fair peer-review system, which is all easy to use. Visit http://www.dovepress.com/ testimonials.php to read real quotes from published authors. 